\title{
Functional Microvascular Anatomy of the Horse Eye: A Scanning Electron Microscopic Study of Corrosion Casts
}

\author{
Hiroyoshi Ninomiya ${ }^{1}$, Tomo Inomata ${ }^{2}$ \\ ${ }^{1}$ Yamazaki Gakuen University, Tokyo, Japan \\ ${ }^{2}$ Department of Laboratory Animal Science, Azabu University, Sagamihara, Japan \\ Email: ${ }^{*}$ ninomiya@azabu-u.ac.jp
}

Received 6 March 2014; revised 6 April 2014; accepted 18 April 2014

Copyright (C) 2014 by authors and Scientific Research Publishing Inc.

This work is licensed under the Creative Commons Attribution International License (CC BY). http://creativecommons.org/licenses/by/4.0/

c) (i) Open Access

\section{Abstract}

Objective: This study presents the microvasculature of the horse iris, ciliary process, retina, and choroid and discusses the functional significance of the vasculature. Procedure: Seven horses were used for this study. The ocular vascular system was injected with methylmethacrylate resin via the carotid artery, and the vascular corrosion casts were observed using a scanning electron microscope. Results: The iridial vessels showed a wavy course. The ciliary process was supplied by 2 arterial routes: the iridial and ciliary arterial circles. The subjects displayed a paurangiotic retina with retinal vessels extending only a short distance around the disc. The retinal arterioles and venules ran in closely related pairs, and the capillaries formed hairpin loops. No central retinal artery was seen in the equine eyes examined. The choriocapillaris in the avascular retina was arranged in honeycomb hexagon lobules and formed a more densely packed network than that in the vascular retina. There were 2 distinct venous drainage systems in the horse choroid: the vortex veins and the posterior ciliary veins. The vortex vein ampulla was flattened and showed a slit-like lumen at the merge site with the ophthalmic vein. The vortex veins demonstrated a marked constriction before leaving the eye. Discussion: The 2 choroidal drainage systems may compensate each other in event of occlusion. The ampulla and the constriction in the vortex veins may act as a valve regulating the blood flow to keep the eye at an optimum size and the intraocular pressure within the normal physiological range.

\section{Keywords}

Corrosion Cast, Eye, Horse, Microvasculature, Scanning Electron Microscopy

\footnotetext{
${ }^{*}$ Corresponding author.
} 


\section{Introduction}

To date, morphological studies of ocular vasculature in the horse have been mostly limited to descriptions of the large vessels to the eyeball [1]-[5]. Anderson et al. reported the vascular anatomy of the equine and canine iris by means of methylmethacrylate resin injection [1]. Smith et al. described the anterior vasculature and aqueous drainage paths in the pony eye by using scanning electron microscopy of corrosion casts [2]. Shaepdrijver et al. described retinal vascular patterns in various animals including the horse with the resin cast technique [3]. Simoens et al. described anatomy of the ocular arteries in the horse [4] and noted the presence of posterior ciliary veins in the horse and bovine [5]. Molleda et al. showed clear fluorescein angiography of the equine ocular fundus [6]. However, these reports did not describe the detail of the microvasculature of the iris, ciliary process, choroid, and retina, which may be useful in clinical diagnosis and experimental work. The horse has a paurangiotic retina in which sparse retinal vessels are found around the optic disc. There are no vessels in the rest of the fundus and the retina is supplied by choroidal vessels, yet the relatively few accounts of the anatomy of the retina and choroid in the horse mention only its most striking features. Morrison et al. using scanning electron microscopy of corrosion casts, described the microvascular anatomy of mammalian ciliary process including that of the rat, guinea pig, cat, dog, sheep, goat, pig, and cow [7], but they did not describe that of the horse. Therefore, the purpose of this study was to describe the microvascular anatomy of the iris, ciliary process, retina, and choroid and to broaden our general understanding of the horse eye. The functional significance of the microvasculature of these organs is also discussed.

\section{Materials and Methods}

Animals: In total, the heads and necks of 7 horses were obtained from a slaughterhouse. The animals were of varying age (4 - 7 years), breed and history and they were subjected to euthanasia for various reasons other than ocular disease. Each horse was decapitated and the cranial blood vessels were flushed with a saline solution through a catheter inserted in the carotid arteries. Perfusion continued until the saline solution emerging from the jugular vein was completely clear of blood. Subsequently the orbital vasculature was filled by the same approach with approximately 200 cc of a combination of methylmethacrylate monomer and Mercox (Dainippon Ink \& Chemical Co. Ltd., Tokyo, Japan) (ratio by volume = 7:3). The orbital blood vessels were manually injected and the injection pressure was $100-120 \mathrm{mmHg}$. After polymerization, the eyes injected with the resin were enucleated and macerated for $1-2$ days by repeated baths in a $20 \% \mathrm{NaOH}$ solution at $50^{\circ} \mathrm{C}$; samples were then rinsed in running water. Each part of the ocular vascular casts was isolated and mounted on an aluminum stub under a dissecting microscope. Samples were then sputtered with gold in an ion-coater (IB-3; Eiko Engineering Co. Ltd., Ibaraki, Japan) and examined using a scanning electron microscope (ABT-32; Topcon Co. Ltd., Tokyo, Japan).

\section{Results}

\subsection{Vasculature Supplying Blood to the Orbit}

The vasculature to the eyeball has been described in detail previously [1]-[5]. Briefly, the external and internal ophthalmic arteries are the major supply to the eye. The former artery enters the orbital cavity after it leaves the alar canal and the latter arises from the internal carotid artery and runs through the orbital fissure with the optic nerve. In the orbital cavity, the external and internal ophthalmic arteries anastomose with one another. The external ophthalmic artery is approximately $1.0 \mathrm{~mm}$ in diameter and follows an anterior course along the optic nerve toward the eyeball. The ophthalmic artery gives rise to 2 long posterior ciliary, the short posterior ciliary, 2 anterior ciliary, and the chorioretinal arteries.

\subsection{Vasculature of the Anterior Segment}

Blood to the anterior segment including the iris and ciliary process is supplied by the long posterior ciliary via two ring arteries. The iridial ring artery circumscribes the root of the iris forming the (major) iridial arterial ring (Figure 1). Branches of the iridial ring artery extend toward either the pupil to supply the capillary net of the iris or the ciliary process. The iridial arteries are relatively thick $(162-188 \mu \mathrm{m}$ in diameter) and follow either a crimped or somewhat coiled course (Figure 2). Capillaries spread to the periphery of the iris and then loop back toward the ciliary process. Occasionally, endothelial protrusions or sphincter-like constrictions occur in the 


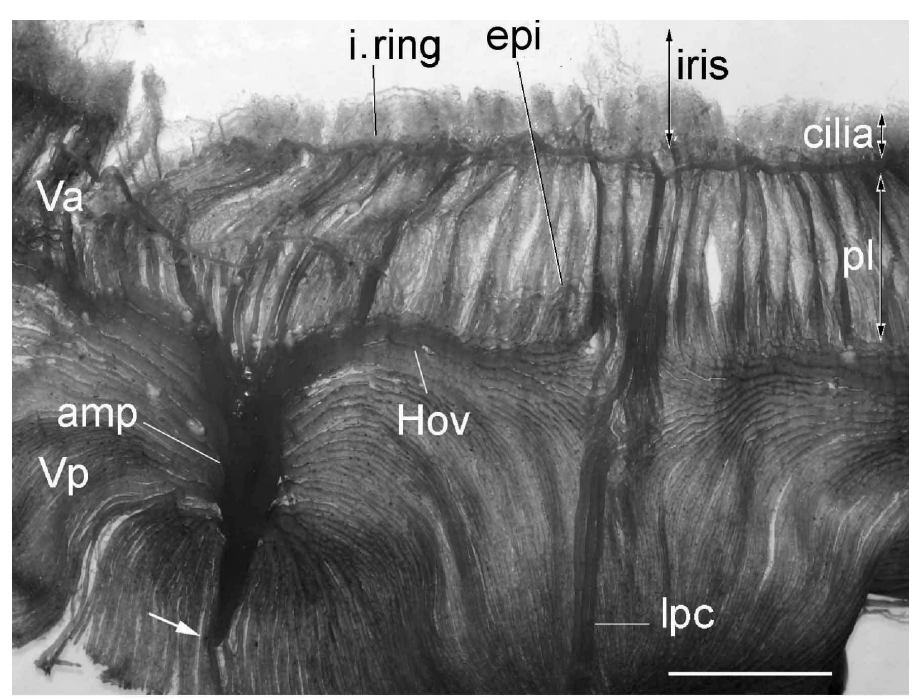

Figure 1. General view of a resin cast of the anterior segment. Iridial vessels are not well injected in this specimen. Note that the vortex vein shows a marked constriction (thick arrow). amp: vortex vein ampulla, cilia: ciliary process, Hov: venous circle of Hovius, i. ring: iridial arterial ring, lpc: long posterior ciliary artery, pl: pars plana, Va: anterior segment of the vortex vein, Vp: posterior segment of the Vortex vein. Bar $=5 \mathrm{~mm}$.

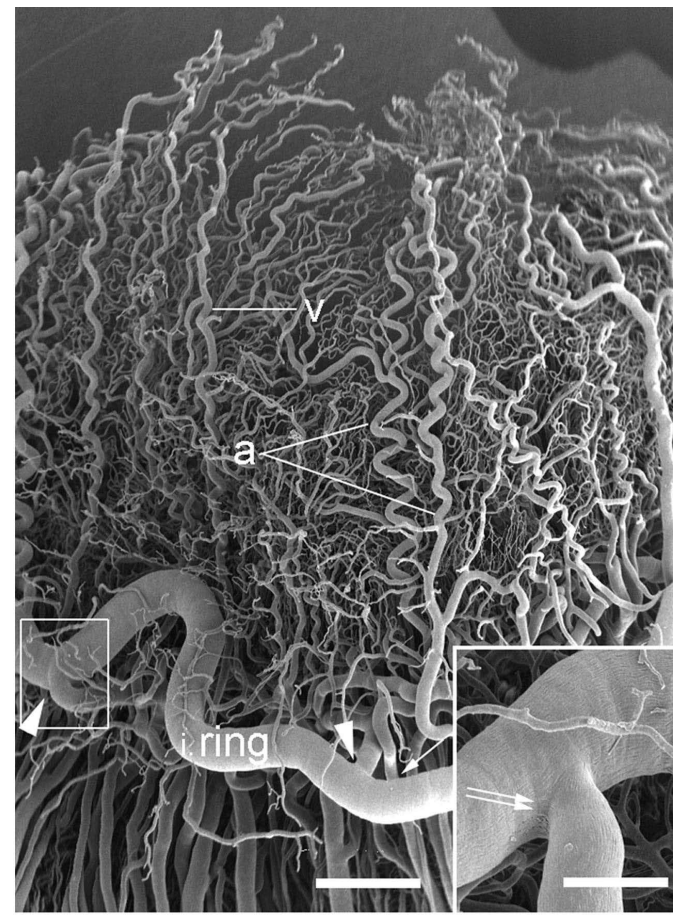

Figure 2. Scanning electron micrograph of the iridial vessels. Iridial vessels show a crimped course and have sphincter-like constrictions at the branching sites (arrow heads) from the iridial arterial ring. Viewed from outside the eye. a: iridial artery, v: iridial vein. Bar $=250 \mu \mathrm{m}$. Inset: Higher magnification of outlined area showing the constriction (double arrow). Bar $=100 \mu \mathrm{m}$. 
iridial and ciliary arterioles at the branching sites from the iridial arterial circle (Figure 2 Inset) as observed in the sheep, pig, goat, and cow [7]. All arterioles arborize within the ciliary process core into a capillary bed that extends to the sides, margin, and head of each ciliary process. These capillaries drain into large veins in the process core that coalesce into the choroidal veins at the iris root and drain into the anterior segments of the vortex veins at the limbus. The microvessels in the ciliary process form radially arranged, closely packed plates of vessels, and consist of the marginal capillaries, the intraprocess capillaries, and the collecting venules. The marginal capillaries exhibit an irregular bore, marked conglomeration, and large diameter (27 - $73 \mu \mathrm{m})$. They drain posteriorly and conjoin with the choriocapillaris in the central region of the pars plana (Figure 3(A) and Figure 3(B)). Capillaries draining major parts of the ciliary process are also relatively thick (19 - $32 \mu \mathrm{m}$ in diameter) (Figure 4(A) and Figure 4(B)) and empty into the anterior segments of the vortex veins via the pars plana venules. The venous blood from the anterior segment including the iris and ciliary process drains into the anterior segments of the vortex veins. The anterior ciliary arteries arise from the ophthalmic artery and run along the rectus muscles. They supply blood to the corneoscleral limbus and bulbar conjunctiva. The terminal branches of the anterior ciliary and the long posterior ciliary arteries anastomose with one another around the conjunctiva.

\subsection{Vasculature of the Posterior Segment}

Retinal vasculature: The blood supply of the retina originates from the chorioretinal artery. This artery divides into several capillaries just before the optic disc. The horse retina is a paurangiotic type in which the retinal vessels extend partially to the retina (Figure 5). The retinal vessels are small, travel in horizontal bands on either side of the optic disc, and pursue a short course forming a butterfly-like vascular network in a limited area around the disc. A small number of shorter vessels also emerge from the 6 and 12 o'clock positions and extend only a short distance from the optic disc. The rest of the retina is avascular and is dependent on the choriocapillaris. Capillaries supplying the retina radiate out from the optic disc and pursue a straight but somewhat curly

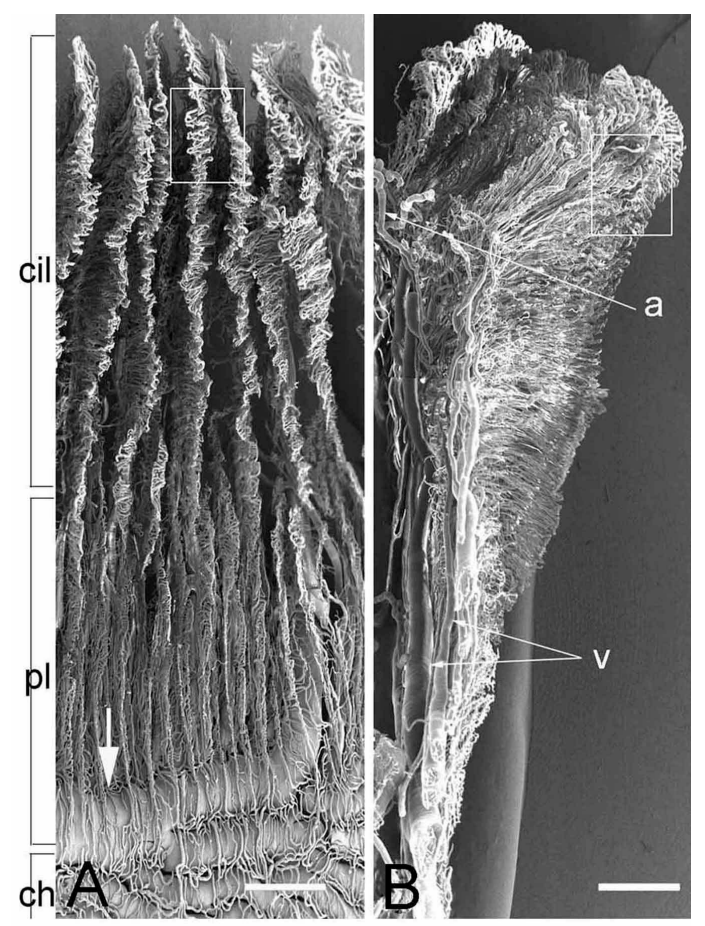

Figure 3. Scanning electron micrograph of vasculature inside the eye showing the ciliary processes (cil) and pars plana (pl). A. Internal view. The venous circle of Hovius (arrow) is seen through the choriocapillaris (ch). Bar $=250 \mu \mathrm{m}$. B. Partially dissected ciliary process demonstrating arteries (a) entering and veins (v) exiting the process. Bar $=250 \mu \mathrm{m}$. 


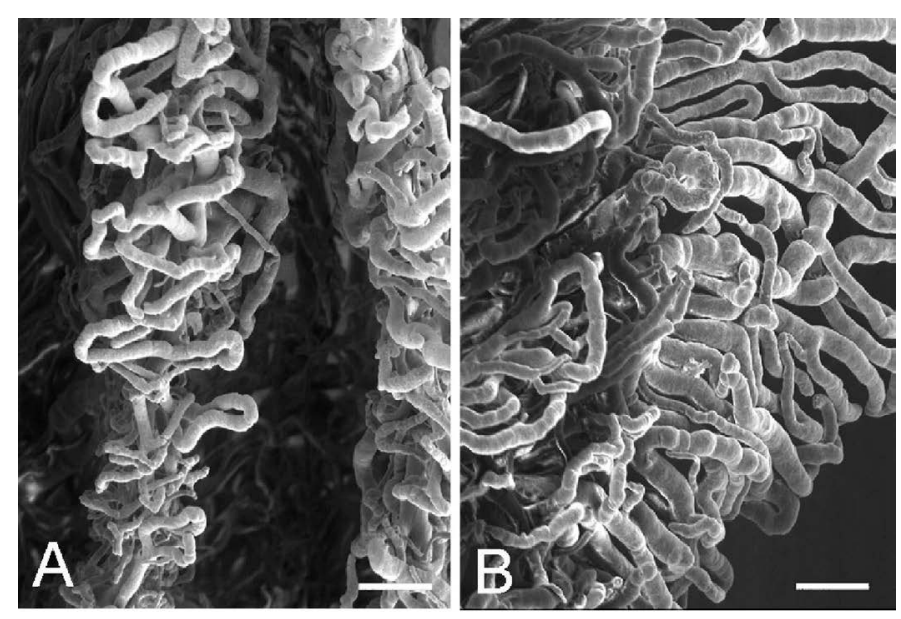

Figure 4. Enlargement of outlined area in Figure 3. A: Marginal capillaries of the ciliary process. $\mathrm{Bar}=100 \mu \mathrm{m}$. B: Capillaries in the ciliary process. $\mathrm{Bar}=100 \mu \mathrm{m}$.

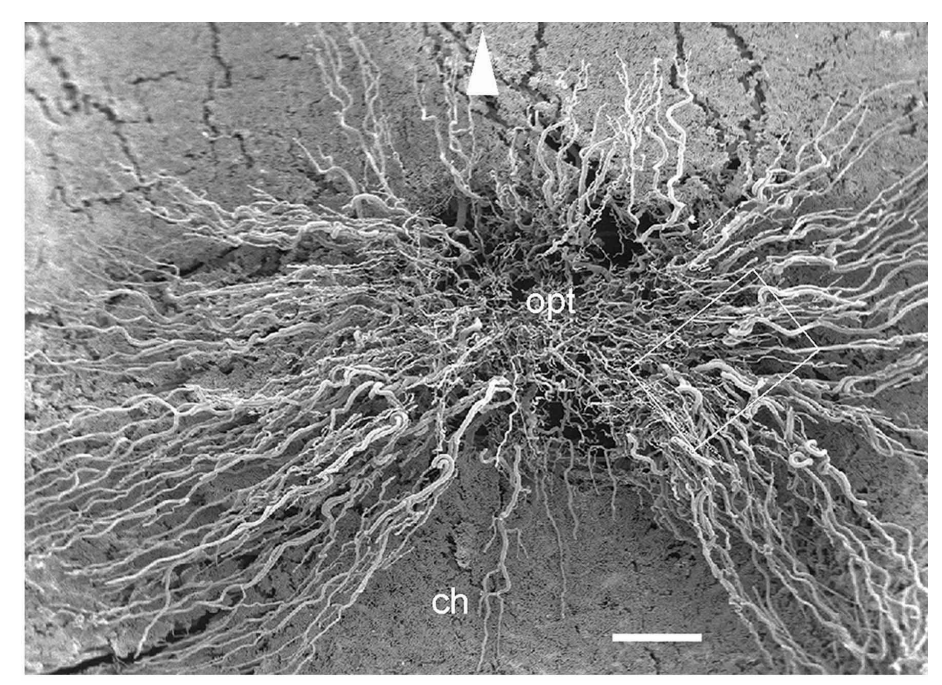

Figure 5. Vascular corrosion cast of the equine fundus. The horse has a paurangiotic retina with fine retinal vessels extending only a short distance around the optic disc (opt). Note the radially arranged retinal vessels emerging from the optic disc. They run in closely related pairs, and capillaries form hairpin loops. Arrow head is at the 12 o'clock position. ch: choriocapillaris. Bar $=250 \mu \mathrm{m}$.

course. The retinal capillaries are relatively thin ( 4 - $5 \mu \mathrm{m}$ in diameter). They give rise to 2 or 3 branches dichotomously, which run parallel to the main capillary. They terminate in small capillaries that form hairpin end loops where the arterial and venous limbs become continuous (Figure 6). The venules are 14 - $20 \mu \mathrm{m}$ in diameter and have a convoluted or extremely undulating course close to the optic disc. The venules then lead up to and drain into the chorioretinal veins.

Choroidal vasculature: The blood supply to the choroid around the optic disc at the posterior pole of the globe originates from the chorioretinal arteries, which give rise to arterioles supplying the retina and the peridiscal choroid. Most of the remaining choroid is supplied by the posterior ciliary arteries. All choroidal arteries run parallel in the vascular lamina of the choroid so as to interdigitate the densely packed choroidal veins draining this region (Figure 1). The arterioles as the afferent vessels penetrate the tapetum at right angles to the tapetal fundus (Figure 7). The arterioles are arranged at regular distances and join the choriocapillaris; the drainage of the choriocapillaris is affected by a single point of collecting venules (postcapillary venules) in 


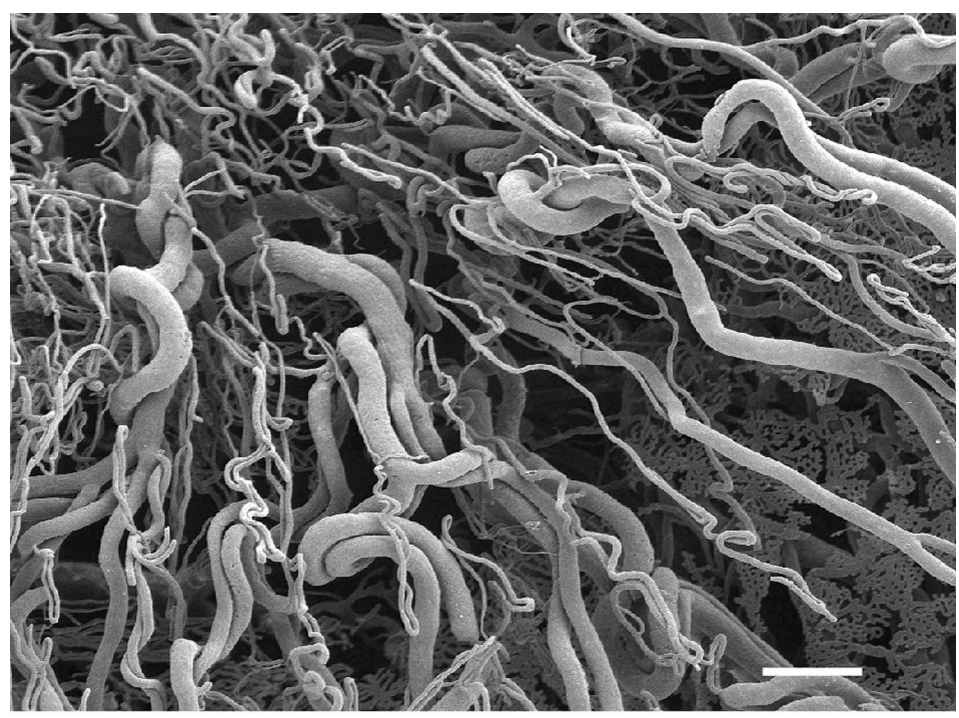

Figure 6. Enlargement of outlined area in Figure 5. Bar $=100 \mu \mathrm{m}$.

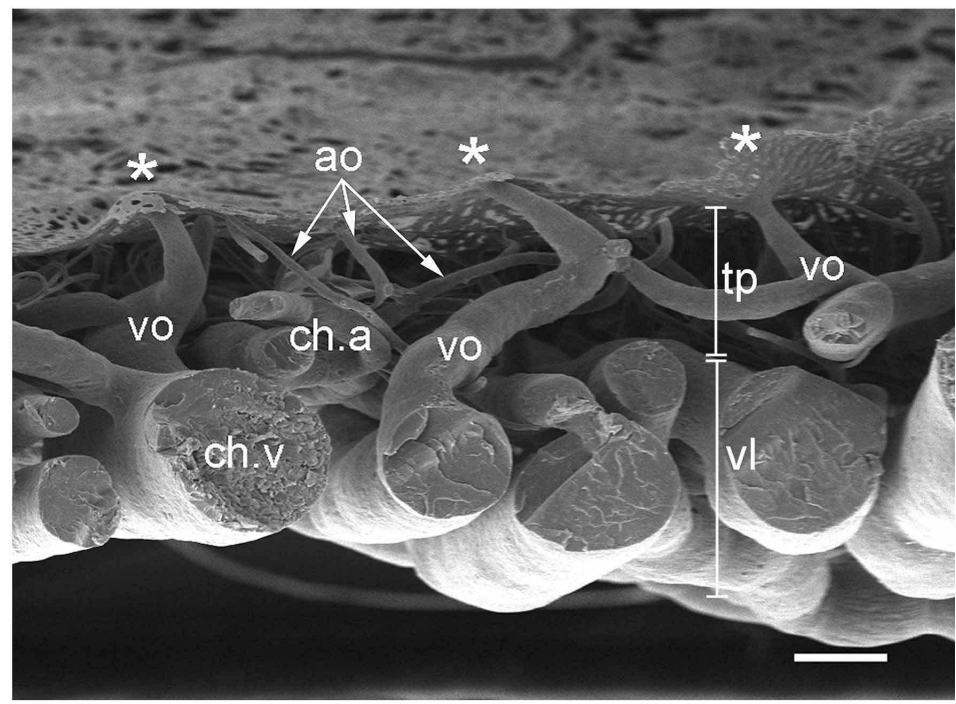

Figure 7. Cross-section of the choroidal vasculature showing choroidal arteries (ch.a) and choroidal veins (ch.v) in the vascular laminar (vl), arterioles (ao) and venules (vo) penetrating the tapetum (tp), and the choriocapillaris (asterisks). Bar $=100 \mu \mathrm{m}$.

the center of each area. The capillaries of the choroid are somewhat flattened, sinusoid-like with a luminal diameter of $16-23 \mu \mathrm{m}$, lie homogeneously distributed in the choroid, and form a very dense freely anastomosing single-layer capillary bed in the choroid. The pattern of the choriocapillaris changes from the peripapillary area with retinal vessels to the peripheral area without retinal vessels. In the area without retinal vessels, the choriocapillaris as a freely communicating network appears as a homogeneous and distinct lobular capillary network of about $200 \mu \mathrm{m}$ in diameter showing a honeycomb or star-shaped hexagon pattern which is recognized as the stars of Winslow [8] (Figure 8, Figure 9(A)). The choriocapillaris is densely packed with a high capillary to inter-capillary ratio. The collecting venule is located in the center of the lobule. In the peripapillary area with retinal vessels, the choriocapillaris appears as a nonhomogenous and nonlobular monolayer capillary network comprising irregular patterns (Figure 9(B)). The choriocapillaris in the 6 and 12 o'clock positions of the optic nerve, where the retinal vessels are quite sparse, also show a nonhomogenous pattern. The venous blood from the peripapillary region drains into the chorioretinal vein. 


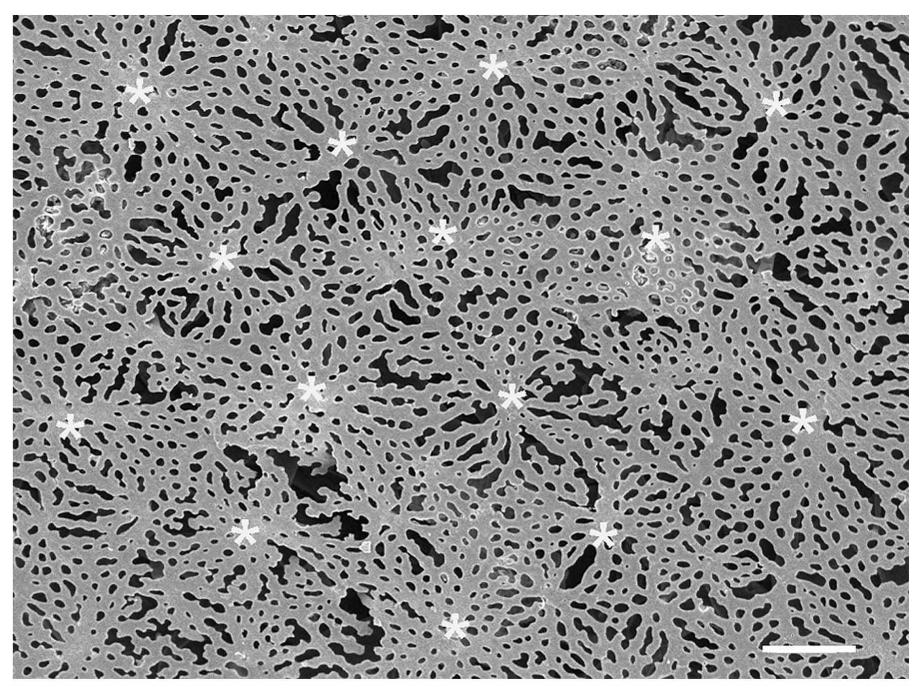

Figure 8. Choriocapillaris in the avascular retina. Viewed from inside the eye. The choriocapillaris is regularly arranged in honeycomb hexagon lobules. Stars of Winslow are clearly seen (asterisks). Bar $=100$ $\mu \mathrm{m}$.

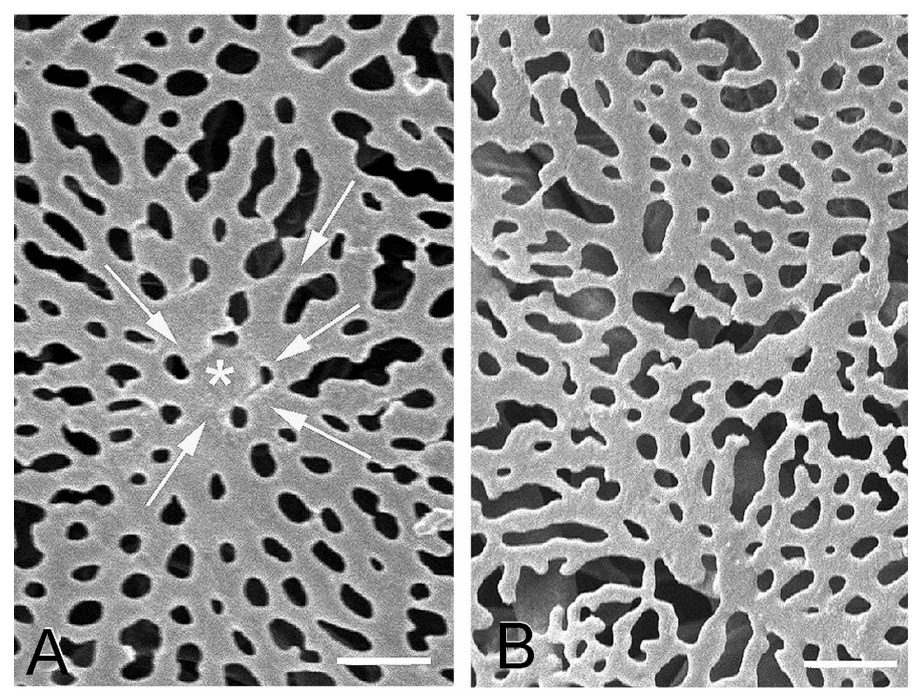

Figure 9. Enlargement of choriocapillaris network. A: Avascular retinal area. Arrows show blood-flow direction. Bar $=25 \mu \mathrm{m}$. B: Vascular retinal area. $\mathrm{Bar}=25 \mu \mathrm{m}$.

\subsection{Venous Drainage of the Eyeball}

Along the outer portion of the iridial ring artery is an interconnecting network of veins in the limbus region known as the intrascleral venous plexus, which receives aqueous humor from the veins that drain the angular aqueous plexus. The plexus then directly drains into the veins of the choroidal venous system (i.e., anterior segments of the vortex veins). The anterior segments of the vortex veins drain blood from the iris and ciliary process. The posterior segments of the vortex veins drain the entire choroid into the circumferential veins known as the circle of Hovius (Figure 1, Figure 3(A)). The venous circle is a complete ring around the eyeball lying anterior to the equator. The circle is drained by 4 vortex veins, which are located on the dorsal, ventral, nasal, and temporal sides of the eyeball near the corneoscleral junction. The vortex veins have an ampulla with a very irregular and flattened bore and pass obliquely through the suprachoroidal space and the sclera just posterior to the equator of the eyeball and exit from the globe (Figure 1, Figures 10(A)-(C)). The veins demonstrate a con- 
spicuous constriction at the merge site with the ophthalmic vein (Figure 1, Figure 10(A) and Figure 10(B)) and follow the curvature of the eyeball within the orbital fossa. As has been reported by Simoens et al. [5], several posterior ciliary veins, which drain the choriocapillaris in the post equatorial area via the posterior segments of the vortex veins, are present. Unlike the vortex veins, the posterior ciliary veins are relatively thin and perforate the sclera somewhat perpendicularly. The choroidal and vortex veins are devoid of valves.

\section{Discussion}

The major contribution of the present study is the demonstration of a detailed three-dimensional view of the microvasculature of the equine retina, choroid, and ciliary process. A number of structure-function relationships are well demonstrated by the corrosion casting technique, and several are noteworthy.

Capillaries of the iris are arranged radially, exhibit a uniform round bore, and undulate, presumably to accommodate changes in the state of the iris. The horse's ciliary process receives blood from the iridial arterial ring as in the sheep, goat, pig, and cow [7]. The ciliary angioarchitecture, comprising separate plates of vessels arranged concentrically, presumably facilitates unrestricted pupillary dilation and constriction. Capillaries supplying the anterior margin of the ciliary process exhibit an irregular bore with marked focal constrictions and are highly complex and somewhat coiled. Additionally, they form thoroughfare channels, draining blood from the ciliary arterioles into the pars plana venules, bypassing the ciliary processes entirely. This bypassing of capillaries may explain the fact that the blood in the pars plana venules is not truly venous blood, but rather highly oxygenated blood [9]. The configuration of the marginal capillaries and the rich blood supply to the ciliary process may affect aqueous humor formation and resorption.

The horse has a predominantly avascular retina, with only a narrow band of retinal vasculature around the optic disc as it is in the guinea pig [3]. Both these animals demonstrate species adaptations in which the oxygen requirement of the inner retina is extremely low compared with that of the outer retina [10]. The retinal vascular network is an unrecognized disturbance to the optical filtering properties of the eye; a photon has a $40 \%-50 \%$ chance of encountering one or more capillaries before it reaches a photoreceptor [11]. The avascular structure of the retina provides a low-scattering passage for light to travel from the retinal surface to the photoreceptors and thus ensures the sharpest vision in birds. The equine eye with retinal avascularity as in the bird may allow for

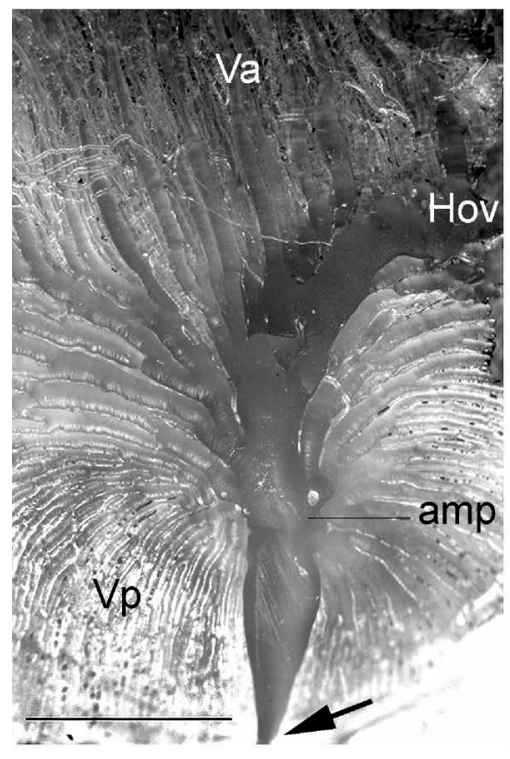

(A)

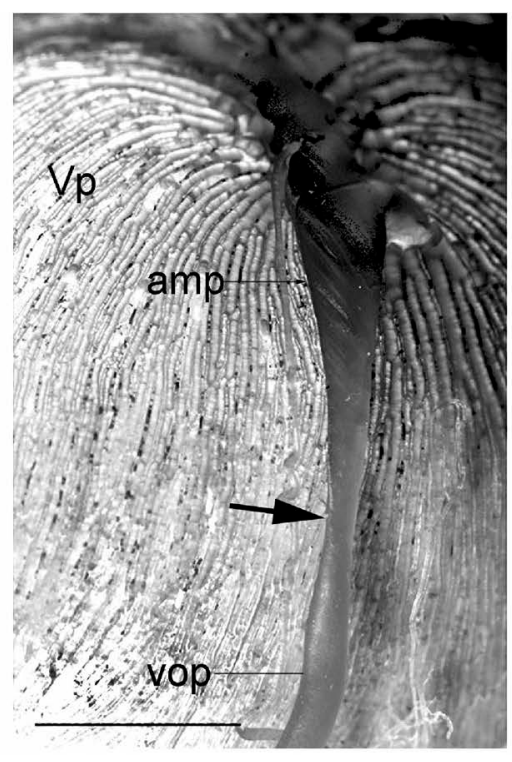

(B)

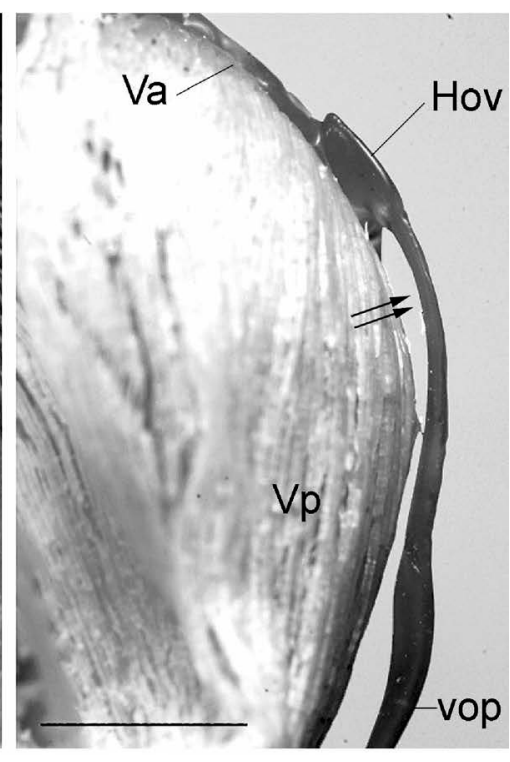

(C)

Figure 10. Vascular cast of a vortex vein. (A) A vortex vein showing the anterior (Va) and posterior (Vp) segments of the vein merging into the venous circle of Hovius (Hov). Note the marked constriction (thick arrow). amp: vortex vein ampulla. Bar = $5 \mathrm{~mm}$; (B) A vortex vein with a constriction (thick arrow) at the merge site with the ophthalmic vein (Vop). amp: vortex vein ampulla, Vp: posterior segment of the vortex vein. Bar $=5 \mathrm{~mm}$; $(\mathrm{C})$ Lateral view of the vortex vein. Note the flattened vortex vein ampulla (double arrow). Hov: the venous circle of Hovius, Va: anterior segment of the vortex vein, Vop: ophthalmic vein. Vp: posterior segment of the vortex vein. Bar $=5 \mathrm{~mm}$. 
capture of photons directly and contribute to the fact that visual acuity has been estimated at 20:30 to 20:60. This means the horse's visual acuity is among the best of the domestic mammals and better than that of many people [12]. Interestingly, only horses and marsupials [13] have the arterial limbs and venous limbs with hairpin loops in the retinal vasculature. Various theories have been proposed to explain the possible functional and physiological basis for this hairpin vascular pattern in the marsupials. The hairpin vascular arrangement may be responsible for thermoregulation [14] or for optimizing retinal oxygen tensions [15]. It has also been speculated that the hairpin arrangement allows the developing retinal vasculature in the fetus to become functionally patent without remodeling [16]. Because these theories are far from conclusive evidence, further follow-up studies are warranted regarding interpretation of the hairpin arrangement in the retinal vasculature.

Comparison of the vascular arrangement of the choriocapillaris in the region with the retinal vessels with that without retinal vessels shows that the latter consists of a more compactly and systematically arranged network with a honeycomb hexagon lobular pattern, which is clearly seen as the stars of Winslow. Because the retina receives oxygen and nutrients from the retinal and choroidal vessels, the rich choriocapillaris may compensate for the absence of retinal vessels to nourish the retina, even though avascular retinas have the potential for anaerobic metabolism [10].

The anterior and posterior chambers are filled with aqueous humor and maintained at an optimum size and pressure by production of this fluid by the ciliary body. The aqueous humor exits the eye either through the trabecular meshwork into Schlemm's canal and aqueous veins (conventional pathway) or through the ciliary muscle, across the sclera, through the suprachoroidal space, and into the choroidal veins (uveoscleral outflow) [17]. High intraocular pressure is caused by decreased outflow of this fluid from the equine eye. This could be due to an abnormally developed, damaged, or obstructed drain. As has been reported by Simoens et al., the horse has 2 drainage channels for the choroid-the vortex and the posterior ciliary veins [5]. The former drains most of the choroid, iris, and ciliary body, while the latter drains limited areas of the choroid posterior to the equator of the eye. In the absence of venous valves in the emissary veins of the choroid, blood flow can occur in either direction depending upon relative pressure and other physical factors. The posterior ciliary veins may be capable of transporting a sizeable portion of the blood flow to the episcleral veins in event of occlusion of the vortex veins, acting as a spillway for the vortex veins. In the rat eye, there are 2 separate drainage pathways: the entire choroid is drained by the posterior ciliary vein, and the anterior segments including the iris, ciliary body, and bulbar conjunctiva are drained by the vortex veins [18]. Rats have been exclusively used for the study of intraocular pressure elevation by cauterization of vortex veins. However, no elevation in intraocular pressure was shown in the studied rats [19]. Presumably, another venous drainage via the posterior ciliary vein may be responsible for preventing elevation of intraocular pressure. Porcine eyes also have venous outflow through the choroid into the vortex veins and posterior ciliary veins [5], and it has been reported that no significant choroidal drainage effects were observed even though the vortex veins were blocked [20]. In contrast to its frequent occurrence in dogs and cats, glaucoma in horses seems to be rare [21]. This low prevalence is difficult to explain in light of the frequency of ocular trauma, corneal perforation, and severe uveitis [21]. It has been suggested that the resistance of the equine eye to the development of glaucoma may be related to the presence of an extensive collateralization of the vascular bed in the pony eye, which prevents reduced aqueous humor drainage even in the case of occlusion of any one vessel [22]. The equine's uniquely wide and well-defined trabecular meshwork with a distinct pectinae ligament makes collapse of the ciliary cleft practically impossible [23] and provides a potentially extensive route of outflow of aqueous humor in the equine eye [24]. Concomitantly, the equine eye has 2 venous channels draining blood from the choroid, the vortex system and the posterior ciliary veins. The equine ocular venous system with 2 venous drainage channels may compensate for occlusion of any one vessel or a small area, with little detrimental effect on aqueous humor drainage, as it is in the rat eye. This morphology of the iridocorneal angle and vascular anatomy may help to explain the rarity of glaucoma in the horse. As these data are far from conclusive more fruitful studies on the causes of glalucoma and its rarity in horses are needed.

The vortex vein ampulla runs obliquely through the sclera and joins the episcleral vein via slit-like lumens at the bulbar equator. Owing to the oblique course through the sclera, the lumen of the ampulla is easily compressed, resulting in fluid retention when swelling of the eyeball compresses the transscleral vessels [25] [26]; this may act as a valve regulating the blood flow to keep the intraocular pressure within the normal physiological range. The uveal effusion syndrome may also be associated with this anatomy of the vortex vein. A similar structure and mechanism occurs at the junction of the ureter and the bladder. On the oblique course of the ureter through the bladder wall, the ureter forms ureterovesical valves to prevent reflux of urine from the bladder [27]. 
The vortex veins display a marked constriction at the merge site with the ophthalmic vein, and this constriction may play a role in controlling venous blood flow from the eyeball.

\section{References}

[1] Anderson, B.G. and Anderson, W.D. (1977) Vasculature of the Equine and Canine Iris. American Journal of Veterinary Research, 38, 1791-1799.

[2] Smith, P., Samuelson, D. and Brooks, D. (1988) Aqueous Drainage Paths in the Equine Eye: Scanning Electron Microscopy of Corrosion Casts. Journal of Morphology, 198, 33-42. http://dx.doi.org/10.1002/jmor.1051980105

[3] De Schaepdrijver, L., Simoens, P., Lauwers, H. and De Geest, J.P. (1989) Retinal Vascular Patterns in Domestic Animals. Research in Veterinary Science, 47, 34-42.

[4] Simoens, P., Muylle, S. and Lauwers, H. (1996) Anatomy of the Ocular Arteries in the Horse. Equine Veterinary Journal, 28, 360-367. http://dx.doi.org/10.1111/j.2042-3306.1996.tb03106.x

[5] Simoens, P., Van den Broeck, W. and Lauwers, H. (2001) Scanning Electron Microscopic Study of the Posterior Ciliary Veins in Domestic Ungulates. Folia Morphologica, 60, 21-26.

[6] Molleda, J.M., Cervantes, I., Galán, A., Tardón, R., Gallardo, J.M. and Martin-Suarez, E.M. (2008) Fluorangiographic Study of the Ocular Fundus in Normal Horses. Veterinary Ophthalmology, 11, 2-7.

http://dx.doi.org/10.1111/j.1463-5224.2008.00621.x

[7] Morrison, J.C., De Frank, M.P. and Buskirk, M.M. (1987) Comparative Microvascular Anatomy of Mammalian Ciliary Processes. Investigative Ophthalmology \& Visual Science, 28, 1325-1340.

[8] Schoster, J.V. (2006) Normal Fundus and Variations in the Dog, Cat and Horse. http://cal.vet.upenn.edu/projects/ophthalmology/ophthalmo_files/Tools/Retina.pdf

[9] Funk, R.H. Wagner, W. and Wild, J. (1992) Microendoscopic Observations of the Hemodynamics in the Rabbit Ciliary Processes. Current Eye Research, 11, 543-551. http://dx.doi.org/10.3109/02713689209001810

[10] Yu, D.Y. and Cringle, S.J. (2001) Oxygen Distribution and Consumption within the Retina in Vascularised and Avascular Retinas and in Animal Models of Retinal Disease. Progress in Retinal and Eye Research, 20, 175-208. http://dx.doi.org/10.1016/S1350-9462(00)00027-6

[11] Snodderly, D.M. and Weinhaus, R.S. (1990) Retinal Vasculature of the Fovea of the Squirrel Monkey, Saimiri sciureus: Three-Dimensional Architecture, Visual Screening, and Relationships to the Neuronal Layers. Journal of Comparative Neurology, 297, 145-163. http://dx.doi.org/10.1002/cne.902970111

[12] Timney, B. and Macuda, T. (2001) Vision and Hearing in Horses. Journal of American Veterinary Medical Association, 218, 1567-1574. http://dx.doi.org/10.2460/javma.2001.218.1567

[13] McMenamin, P.G. (2007) The Unique Paired Retinal Vascular Pattern in Marsupials: Structural, Functional and Evolutionary Perspectives Based on Observations in a Range of Species. British Journal of Ophthalmology, 91, 1399-1405. http://dx.doi.org/10.1136/bjo.2007.119537

[14] Walls, G.L. (1939) Notes on the Retinae of Two Opossum Genera. Journal of Morphology, 64, 67-87. http://dx.doi.org/10.1002/jmor.1050640106

[15] Craigie, E.H. (1945) The Architecture of the Cerebral Capillary Bed. Biological Review, 146, 20133-20146.

[16] Chase, J. (1982) The Evolution of Retinal Vascularization in Mammals. A Comparison of Vascular and Avascular Retinae. Ophthalmology, 89, 1518-1525. http://dx.doi.org/10.1016/S0161-6420(82)34608-4

[17] Fautsch, M.P. and Johnson, D.H. (2006) The Second ARVO/Pfizer Research Institute Working Group. Aqueous Humor Outflow: What Do We Know? Where Will It Lead Us? Investigative Ophthalmology \& Visual Science, 47, 41814187. http://dx.doi.org/10.1167/iovs.06-0830

[18] Bhutto, I.A. and Amemiya, T. (2001) Microvascular Architecture of the Rat Choroid: Corrosion Cast Study. Anatomical Record, 264, 63-71. http://dx.doi.org/10.1002/ar.1102

[19] Grozdanic, S.D., Betts, D.M., Sakaguchi, D.S., Kwon, Y.H., Kardon, R.H. and Sonea. I.M. (2003) Temporary Elevation of the Intraocular Pressure by Cauterization of Vortex and Episcleral Veins in Rats Causes Functional Deficits in the Retinal and Optic Nerve. Experimental Eye Research, 77, 27-33. http://dx.doi.org/10.1016/S0014-4835(03)00089-7

[20] Wagner, J.A., Edwards, A. and Schuman, J.S. (2004) Characterization of Uveoscleral Outflow Enucleated Porcine Eyes Perfused under Constant Pressure. Investigative Ophthalmology \& Visual Science, 45, 3203-3206. http://dx.doi.org/10.1167/iovs.03-1176

[21] Wilcock, B.P., Brooks, D. and Latimer, A. (1991) Brief Communications and Case Reports: Glaucoma in Horses. Veterinary Pathology, 28, 74-78. http://dx.doi.org/10.1177/030098589102800110

[22] Smith, P.J., Samuelson, D.A., Brooks, D.E. and Whitley, R.D. (1986) Unconventional Aqueous Humor Outflow of 
Microspheres Perfuse into the Equine Eye. American Journal of Veterinary Research, 47, 2445-2453.

[23] De Geest, J.P., Lauwers, H., Simoens, P. and De Schaepdrijver, L. (1990) The Morphology of the Equine Iridocorneal Angle: A Light and Scanning Electron Microscopic Study. Equine Veterinary Journal, 10, 30-35.

[24] Samuelson, D., Smith, P. and Brooks, D. (1989) Morphologic Features of the Aqueous Humor Drainage Pathways in Horses. American Journal of Veterinary Research, 50, 720-727.

[25] Norn, M. (2005) The Glaucoma Theory of CF Heerfordt. Dan Medicinhist Arbog, 33, 103-114.

[26] Elagouz, M., Stanescu-Segall, D. and Jackson, T.L. (2010) Uveal Effusion Syndrome. Survey of Ophthalmology, 55, 134-145. http://dx.doi.org/10.1016/j.survophthal.2009.05.003

[27] Viana, R., Batourina, E., Huang, H., Dressler, G.R., Kobayashi, A., Behringer, R.R., Ellen Shapiro, E., Hensle, T., Lambert, S. and Mendelsohn, C. (2007) The Development of the Bladder Trigone, the Center of the Anti-Reflux Mechanism. Development, 134, 3763-3769. http://dx.doi.org/10.1242/dev.011270 